\title{
ON THE TELEGRAPH EQUATION WITH A SMALL PARAMETER
}

J. Kisyński, Lublin University of Technology, Lublin, Poland, jan.kisynski@gmail.com

This work is an edited translation, by A. Bobrowski and W. Chojnacki, of the original Polish text entitled $O$ równaniu telegrafistów z matym parametrem which appeared in Prace Naukowe Politechniki Lubelskiej 212, Matematyka (Research Bulletin of the Lublin University of Technology 212, Mathematics), 1991, vol. 12, pp. 17-40.

\section{Introduction}

In this paper we will be concerned with the telegraph equation

$$
a \frac{\partial^{2} u}{\partial t^{2}}+b \frac{\partial u}{\partial t}+c u=\frac{\partial^{2} u}{\partial x^{2}}
$$

where $a>0, b>0$ and $c \geq 0$. We will be interested in studying the behaviour of solutions of this equation for $t \geq 0$ in the case where the coefficient $a$ is small. In particular, our aim will be to estimate the difference between solutions of $(\mathrm{T})$ and solutions of the diffusion equation

$$
b \frac{\partial u}{\partial t}+c u=\frac{\partial^{2} u}{\partial x^{2}} .
$$

Such estimates have been studied by a number of authors, including M. Zlámal [11-13], J. Kisyński [2,3], J. Smoller [7], J. Nedoma [6], and M. Sova [9]. The paper by Zlámal [11] was inspired by a very specific technical problem. The question of estimating the difference between solutions of $(\mathrm{T})$ and $(\mathrm{D})$ has also been considered in the context of neutron transport, see A.M. Weinberg and E.P. Wigner [10, p. 235] and W. Baran [1].

It turned out that it is fruitful to treat $(\mathrm{T})$ as a particular case of a certain type of differential equations in Banach spaces. For, such an approach allows uniform, systematic treatment of a number of boundary-value problems for $(\mathrm{T})$ and obtaining in all these cases the same estimates in terms of the norms of underlying Banach spaces in which the problem is well-posed. We stress here that boundary-value problems that fit into our framework may include the solution $u$ and its spatial derivative $\frac{\partial u}{\partial x}$, but not its time derivative $\frac{\partial u}{\partial t}$.

The difference between the results we present here and those obtained in [3] lies in the fact that now we are able to consider the case of non-zero $c$. This is important for applications e.g. in neuron transport theory. To treat $c>0$, we introduce a new operatorvalued function which we denote by $V(t, a, b, c, A)$. This function will critically intervene in what we will call the comparison theorem (see [3, p. 372] and Section 3 in the present paper). 


\section{Abstract Telegraph Equation}

Let $E_{0}$ be a Banach space, let $\xi(t),-\infty<t<\infty$, be a strongly continuous cosine operator function with values in the space $\mathcal{L}\left(E_{0}, E_{0}\right)$, and let $A$ be the infinitesimal generator of this cosine operator function, see M. Sova [8] or J. Kisyński [4]. Let

$$
\begin{gathered}
E_{1}=\left\{u \in E_{0}: \text { the function }(-\infty, \infty) \ni t \mapsto \xi(t) u \in E_{0} \text { is of class } C^{1}\right. \text { in } \\
\text { the norm of } \left.E_{0}\right\} .
\end{gathered}
$$

When equipped with the norm

$$
\|u\|_{E_{1}}=\|u\|_{E_{0}}+\sup _{0 \leq 1 \leq t}\left\|\frac{d \xi(t) u}{d t}\right\|_{E_{0}},
$$

$E_{1}$ is a Banach space; see [4, p. 98]. Let us consider the Cartesian product $E_{1} \times E_{0}$ and let us agree to denote its elements as column-vectors $\left(\begin{array}{l}u \\ v\end{array}\right), u \in E_{1}, v \in E_{0}$. Let $k$ be a positive constant. Then the operators

$$
G(t)=\left(\begin{array}{cc}
\xi(k t) & \int_{0}^{t} \xi(k \tau) \mathrm{d} \tau \\
\frac{\mathrm{d} \xi(k t)}{\mathrm{d} t} & \xi(k t)
\end{array}\right), \quad-\infty<t<\infty
$$

belong to $\mathcal{L}\left(E_{1} \times E_{0}, E_{1} \times E_{0}\right)$ and form a one-parameter strongly continuous group with generator

$$
B=\left(\begin{array}{cc}
0 & 1 \\
k^{2} A & 0
\end{array}\right), \quad D(B)=D(A) \times E_{1}
$$

see [4, p. 98]. Now, let $a, b$ and $c$ be fixed scalars with $a>0$. By the Dyson-Phillips bounded perturbation theorem, the operator

$$
B_{a, b, c}=\left(\begin{array}{cc}
0 & 1 \\
a^{-1}(A-c) & -a^{-1} b
\end{array}\right)=\left(\begin{array}{cc}
0 & 1 \\
a^{-1} A & 0
\end{array}\right)-\left(\begin{array}{cc}
0 & 0 \\
a^{-1} c & a^{-1} b
\end{array}\right)
$$

generates a strongly continuous one parameter group of operators in $E_{1} \times E_{0}$. Let us express the operators in this group in the form of operator-valued matrices

$$
\exp \left(t B_{a, b, c}\right)=\left(\begin{array}{ll}
S_{00}(t, a, b, c, A) & S_{01}(t, a, b, c, A) \\
S_{10}(t, a, b, c, A) & S_{11}(t, a, b, c, A)
\end{array}\right)
$$

Then the $S_{i j}(t, a, b, c, A)$ are strongly continuous functions of $t$ with values in $\mathcal{L}\left(E_{1-j}, E_{1-i}\right)$. It is easy to see that the Cauchy problem

$$
\left\{\begin{aligned}
a \frac{\mathrm{d}^{2} u}{\mathrm{~d} t^{2}}+b \frac{\mathrm{d} u}{\mathrm{~d} t}+c u & =A u, \quad-\infty<t<\infty, \\
u(0) & =u_{0} \\
\frac{\mathrm{d} u}{\mathrm{~d} t}(0) & =u_{1}
\end{aligned}\right.
$$

has, for any pair of initial conditions $u_{0} \in \mathcal{D}(A)$ and $u_{1} \in E_{1}$, a $C^{2}\left(-\infty, \infty ; E_{0}\right)$-class solution $u(t)$ with values in $\mathcal{D}(A)$, given by the formula

$$
u(t)=S_{00}(t, a, b, c, A) u_{0}+S_{01}(t, a, b, c, A) u_{1} .
$$


This solution is unique in the class $C^{2}\left(0, T ; E_{0}\right)$ on each interval $(0, T)$. To prove this, suppose that $v(t)$ is another solution of $\left(\mathrm{T}^{*}\right)$. Then, for any arbitrarily fixed $t \in(0, T)$,

$$
v(t)-u(t)=\left.\left[S_{00}(t-\tau) v(\tau)+S_{01}(t-\tau) \frac{\mathrm{d} v(\tau)}{\mathrm{d} \tau}\right]\right|_{\tau=0} ^{\tau=t} .
$$

Using formulae (3) and (4), given in Section 3, for the derivatives of $S_{00}$ and $S_{11}$, one may show that the derivative with respect to $\tau$ of the expression in the brackets is zero, and this implies that $v(t)=u(t)$. Another proof of uniqueness may be obtained by eliminating the first derivative in the differential equation from $\left(\mathrm{T}^{*}\right)$ with the substitution $u(t)=\mathrm{e}^{-\frac{b}{2 a} t} v(t)$ and thereby reducing the problem to the case considered in [4, p. 96].

The operator-valued functions $S_{i j}$ were build from the cosine function $\xi(t)$ via the group $G(t)$ by applying the Dyson-Phillips bounded perturbation theorem. One can show, and this will be done below, that if $b^{2}>4 a c$ (a condition which henceforth will be tacitly assumed), then

$$
S_{01}(t, a, b, c, A)=\mathrm{e}^{-\frac{b}{2 a} t} \int_{0}^{t} J_{0}\left(i \frac{\sqrt{\Delta}}{2 a} \sqrt{t^{2}-\tau^{2}}\right) \xi\left(\frac{\tau}{\sqrt{a}}\right) \mathrm{d} \tau,
$$

where $\Delta=b^{2}-4 a c$ and $J_{0}(i x)$ is the Bessel function of type zero with purely imaginary argument, given by the series

$$
J_{0}(i x)=\sum_{k=0}^{\infty} \frac{\left(\frac{1}{2} x\right)^{2 k}}{(k !)^{2}} .
$$

An immediate consequence of equality (1) and formula (4) from Section 3 is

$$
\begin{gathered}
S_{00}(t, a, b, c, A)=\left(\frac{\mathrm{d}}{\mathrm{d} t}+\frac{b}{a}\right) S_{01}(t, a, b, c, A)= \\
=\mathrm{e}^{-\frac{b}{2 a} t} \xi\left(\frac{t}{2 a}\right)+\mathrm{e}^{-\frac{b}{2 a} t} \int_{0}^{t}\left(\frac{\partial}{\partial t}+\frac{b}{2 a}\right) J_{0}\left(i \frac{\sqrt{\Delta}}{2 a} \sqrt{t^{2}-\tau^{2}}\right) \xi\left(\frac{\tau}{\sqrt{a}}\right) \mathrm{d} \tau .
\end{gathered}
$$

Hence, we see that the solution to $\left(\mathrm{T}^{*}\right)$ is given by the formula

$$
\begin{gathered}
u(t)=\mathrm{e}^{-\frac{b}{2 a} t} \xi\left(\frac{t}{\sqrt{a}}\right) u_{0}+\mathrm{e}^{-\frac{b}{2 a} t} \int_{0}^{t}\left(\frac{\partial}{\partial t}+\frac{b}{2 a}\right) J_{0}\left(i \frac{\sqrt{\Delta}}{2 a} \sqrt{t^{2}-\tau^{2}}\right) \xi\left(\frac{\tau}{\sqrt{a}}\right) u_{0} \mathrm{~d} \tau+ \\
+\mathrm{e}^{-\frac{b}{2 a}} t \int_{0}^{t} J_{0}\left(i \frac{\sqrt{\Delta}}{2 a} \sqrt{t^{2}-\tau^{2}}\right) \xi\left(\frac{\tau}{\sqrt{a}}\right) u_{1} \mathrm{~d} \tau .
\end{gathered}
$$

We now prove (1) by verifying that the right-hand side of the formula is a solution of an appropriate uniquely solvable Cauchy problem. For $u_{1} \in E_{1}$, we have

$$
\left\{\begin{aligned}
\left(a \frac{\mathrm{d}^{2}}{\mathrm{~d} t^{2}}+b \frac{\mathrm{d}}{\mathrm{d} t}+c\right) S_{01}(t, a, b, c, A) u_{1} & =A S_{01}(t, a, b, c, A) u_{1}, \\
& S_{01}(0, a, b, c, A) u_{1}=0, \\
\left.\frac{\mathrm{d}}{\mathrm{d} t}\right|_{t=0} S_{01}(t, a, b, c, A) u_{1} & =u_{1},
\end{aligned}\right.
$$


and, as explained above, this system has a unique solution. Let

$$
K(t, \tau)=\mathrm{e}^{-\frac{b}{2 a} t} J_{0}\left(i \frac{\sqrt{\Delta}}{2 a} \sqrt{\tau^{2}-t^{2}}\right) .
$$

Now, all we need is to check that the integral

$$
\tilde{S}_{01}(t) u_{1}=\int_{0}^{t} K(t, \tau) \xi\left(\frac{\tau}{\sqrt{a}}\right) u_{1} \mathrm{~d} \tau
$$

satisfies $(*)$. The condition $\tilde{S}_{01}(0) u_{1}=0$ is obviously met. Moreover,

$$
\begin{gathered}
\frac{\mathrm{d}}{\mathrm{d} t} \tilde{S}_{01}(t) u_{1}=K(t, t) \xi\left(\frac{t}{\sqrt{a}}\right) u_{1}+\int_{0}^{t} \frac{\partial K}{\partial t}(t, \tau) \xi\left(\frac{\tau}{\sqrt{a}}\right) u_{1} \mathrm{~d} \tau= \\
=\mathrm{e}^{-\frac{b}{2 a} t} \xi\left(\frac{t}{\sqrt{a}}\right) u_{1}+\int_{0}^{t} \frac{\partial K}{\partial t}(t, \tau) \xi\left(\frac{\tau}{\sqrt{a}}\right) u_{1} \mathrm{~d} \tau,
\end{gathered}
$$

and so $\left.\frac{\mathrm{d}}{\mathrm{d} t}\right|_{t=0} S_{01}(t) u_{1}=u_{1}$. We are thus left with checking that the differential equation in system $(*)$ holds. To this end, we calculate

$$
\begin{gathered}
\frac{\mathrm{d}^{2}}{\mathrm{~d} t^{2}} \tilde{S}_{01}(t) u_{1}=-\frac{b}{2 a} \mathrm{e}^{-\frac{b}{2 a} t} \xi\left(\frac{t}{\sqrt{a}}\right) u_{1}+\mathrm{e}^{-\frac{b}{2 a} t} \frac{\mathrm{d}}{\mathrm{d} t} \xi\left(\frac{t}{\sqrt{a}}\right) u_{1}+ \\
+\frac{\partial K}{\partial t}(t, t) \xi\left(\frac{t}{\sqrt{a}}\right) u_{1}+\int_{0}^{t} \frac{\partial^{2} K}{\partial t^{2}}(t, t) \xi\left(\frac{t}{\sqrt{a}}\right) u_{1} \mathrm{~d} \tau
\end{gathered}
$$

and find that

$$
\begin{gathered}
\left(a \frac{\mathrm{d}^{2}}{\mathrm{~d} t^{2}}+b \frac{\mathrm{d}}{\mathrm{d} t}+c-A\right) \tilde{S}_{01}(t) u_{1}=\left[\frac{b}{2} \mathrm{e}^{-\frac{b}{2 a} t}+\frac{\partial K}{\partial t}(t, t)\right] \xi\left(\frac{t}{\sqrt{a}}\right) u_{1}+a \mathrm{e}^{-\frac{b}{2 a}} t \frac{\mathrm{d}}{\mathrm{d} t} \xi\left(\frac{t}{\sqrt{a}}\right) u_{1}+ \\
+\int_{0}^{t}\left(a \frac{\partial^{2}}{\partial t^{2}}+b \frac{\partial}{\partial \tau}+c\right) K(t, \tau) \xi\left(\frac{\tau}{\sqrt{a}}\right) u_{1} \mathrm{~d} t-\int_{0}^{1} K(t, \tau) A \xi\left(\frac{\tau}{\sqrt{a}}\right) u_{1} \mathrm{~d} \tau
\end{gathered}
$$

The last integral in this formula can be transformed by means of integration by parts as follows:

$$
\begin{gathered}
\int_{0}^{t} K(t, \tau) A \xi\left(\frac{\tau}{\sqrt{a}}\right) u_{1} \mathrm{~d} \tau=a \int_{0}^{t} K(t, \tau) \frac{\mathrm{d}^{2}}{\mathrm{~d} \tau^{2}} \xi\left(\frac{\tau}{\sqrt{a}}\right) u_{1} \mathrm{~d} \tau= \\
=\left.a K(t, \tau) \frac{\mathrm{d}}{\mathrm{d} \tau} \xi\left(\frac{\tau}{\sqrt{a}}\right) u_{1}\right|_{\tau=0} ^{\tau=t}-a \int_{0}^{t} \frac{\partial K}{\partial \tau}(t, \tau) \frac{\mathrm{d}}{\mathrm{d} \tau} \xi\left(\frac{\tau}{\sqrt{a}}\right) u_{1} \mathrm{~d} \tau= \\
=a K(t, t) \frac{\mathrm{d}}{\mathrm{d} t} \xi\left(\frac{t}{\sqrt{a}}\right) u_{1}-\left.a \frac{\partial K}{\partial \tau}(t, \tau) \xi\left(\frac{\tau}{\sqrt{a}}\right) u_{1}\right|_{\tau=0} ^{\tau=t}+a \int_{0}^{t} \frac{\partial^{2} K}{\partial \tau^{2}}(t, \tau) \xi\left(\frac{\tau}{\sqrt{a}}\right) u_{1} \mathrm{~d} \tau .
\end{gathered}
$$


Since $\frac{\partial K}{\partial \tau}(t, 0)=0$, we thus obtain

$$
\begin{gathered}
\left(a \frac{\mathrm{d}^{2}}{\mathrm{~d} t^{2}}+b \frac{\mathrm{d}}{\mathrm{d} t}+c-A\right) \tilde{S}_{01}(t) u_{1}=a\left[\frac{b}{2 a} \mathrm{e}^{-\frac{b}{2 a} t}+\frac{\partial K}{\partial t}(t, t)+\frac{\partial K}{\partial \tau}(t, t)\right] \xi\left(\frac{t}{\sqrt{a}}\right) u_{1}+ \\
+\int_{0}^{t}\left[a \frac{\partial^{2} K}{\partial t^{2}}(t, \tau)-a \frac{\partial^{2} K}{\partial \tau^{2}}(t, \tau)+b \frac{\partial K}{\partial t}(t, \tau)+c K(t, \tau)\right] \xi\left(\frac{\tau}{\sqrt{a}}\right) u_{1} \mathrm{~d} \tau= \\
\quad=\int_{0}^{t}\left[a \frac{\partial^{2} K}{\partial t^{2}}(t, \tau)-a \frac{\partial^{2} K}{\partial \tau^{2}}(t, \tau)+b \frac{\partial K}{\partial t}(t, \tau)+c K(t, \tau)\right] \xi\left(\frac{\tau}{\sqrt{a}}\right) u_{1} \mathrm{~d} \tau .
\end{gathered}
$$

It is now clear that the proof will be complete once we show that

$$
a \frac{\partial^{2} K}{\partial t^{2}}(t, \tau)-a \frac{\partial^{2} K}{\partial t^{2}}(t, \tau)+b \frac{\partial K}{\partial t}(t, \tau)+c K(t, \tau)=0
$$

for $0 \leq \tau \leq t$. Let $K_{0}(t, \tau)=J_{0}\left(i \frac{\sqrt{\Delta}}{2 a} \sqrt{t^{2}-\tau^{2}}\right)$. Then we have $K(t, \tau)=\mathrm{e}^{-\frac{b}{2 a} t} K_{0}(t, \tau)$, and equation $\left(\begin{array}{l}* \\ *\end{array}\right)$ takes the equivalent form

$$
\left[a\left(\frac{\partial}{\partial t}-\frac{b}{2 a}\right)^{2}-a \frac{\partial^{2}}{\partial \tau^{2}}+b\left(\frac{\partial}{\partial t}-\frac{b}{2 a}\right)+c\right] K_{0}(t, \tau)=0
$$

which is the same as

$$
a\left[\frac{\partial^{2}}{\partial t^{2}}-\frac{\partial^{2}}{\partial \tau^{2}}-\frac{b^{2}-4 a c}{4 a^{2}}\right] K_{0}(t, \tau)=0
$$

or

$$
\left(\frac{\partial^{2}}{\partial t^{2}}-\frac{\partial^{2}}{\partial \tau^{2}}-\frac{\Delta}{4 a^{2}}\right) K_{0}(t, \tau)=0
$$

Given that

$$
\begin{aligned}
& \frac{\partial^{2} K_{0}}{\partial t^{2}}(t, \tau)=\left.\left(\frac{\sqrt{\Delta}}{2 a} \frac{t}{\sqrt{t^{2}-\tau^{2}}}\right)^{2} \frac{\mathrm{d}^{2}}{\mathrm{~d} x^{2}}\right|_{x=\frac{\sqrt{\Delta}}{2 a} \sqrt{t^{2}-\tau^{2}}} J_{0}(i x)+ \\
& +\left.\frac{\sqrt{\Delta}}{2 a}\left(\frac{1}{\sqrt{t^{2}-\tau^{2}}}-\frac{t^{2}}{\left(\sqrt{t^{2}-\tau^{2}}\right)^{3}}\right) \frac{\mathrm{d}}{\mathrm{d} x}\right|_{x=\frac{\sqrt{\Delta}}{2 a} \sqrt{t^{2}-\tau^{2}}} J_{0}(i x)
\end{aligned}
$$

and

$$
\begin{aligned}
& \frac{\partial^{2} K_{0}}{\partial \tau^{2}}(t, \tau)=\left.\left(\frac{\sqrt{\Delta}}{2 a} \frac{\tau}{\sqrt{t^{2}-\tau^{2}}}\right)^{2} \frac{\mathrm{d}^{2}}{\mathrm{~d} x^{2}}\right|_{x=\frac{\sqrt{\Delta}}{2 a} \sqrt{t^{2}-\tau^{2}}} J_{0}(i x)+ \\
& +\left.\frac{\sqrt{\Delta}}{2 a}\left(\frac{-1}{\sqrt{t^{2}-\tau^{2}}}-\frac{\tau^{2}}{\left(\sqrt{t^{2}-\tau^{2}}\right)^{3}}\right) \frac{\mathrm{d}}{\mathrm{d} x}\right|_{x=\frac{\sqrt{\Delta}}{2 a} \sqrt{t^{2}-\tau^{2}}} J_{0}(i x)
\end{aligned}
$$

we see that equation $\left(\begin{array}{c}* \\ * *\end{array}\right)$ is equivalent to

$$
\left(\frac{\mathrm{d}^{2}}{\mathrm{~d} x^{2}}+\frac{1}{x} \frac{\mathrm{d}}{\mathrm{d} x}-1\right) J_{0}(i x)=0
$$


and that the latter equation holds can be checked immediately by differentiating the series

$$
J_{0}(i x)=\sum_{k=0}^{\infty} \frac{\left(\frac{1}{2} x\right)^{2 k}}{(k !)^{2}}
$$

term by term.

\section{Abstract Diffusion Equation}

Along with the second order problem $\left(\mathrm{T}^{*}\right)$ we consider the initial value problem of order one

$$
\left\{\begin{aligned}
b \frac{\mathrm{d} u}{\mathrm{~d} t}+c u & =A u, \quad t \geq 0 \\
u(0) & =u_{0}
\end{aligned}\right.
$$

The solution of this problem is

$$
u(t)=\mathrm{e}^{-\frac{c}{b} t} \exp \left(\frac{t}{b} A\right)
$$

where $\exp (t A), t \geq 0$, is the one-parameter semigroup of operators in the space $E_{0}$ generated by $A$. This semigroup may be simply expressed in terms of the cosine family from the previous section, namely,

$$
\exp (t A)=\frac{1}{2 \sqrt{\pi t}} \int_{-\infty}^{\infty} \mathrm{e}^{-\frac{\tau^{2}}{4 t}} \xi(\tau) \mathrm{d} \tau, \quad t>0
$$

see J. Kisyński [5, p. 9].

\section{Reducing the Problem of Estimating the Difference Between Solutions of Problems $\left(\mathrm{T}^{*}\right)$ and $\left(\mathrm{D}^{*}\right)$ to a Comparison Theorem}

From $\exp \left(t B_{a, b, c}\right) D\left(B_{a, b, c}\right)=D\left(B_{a, b, c}\right)$ and

$$
\frac{\mathrm{d}}{\mathrm{d} t} \exp \left(t B_{a, b, c}\right)\left(\begin{array}{l}
u_{0} \\
u_{1}
\end{array}\right)=B_{a, b, c} \exp \left(t B_{a, b, c}\right)\left(\begin{array}{l}
u_{0} \\
u_{1}
\end{array}\right)=\exp \left(t B_{a, b, c}\right) B_{a, b, c}\left(\begin{array}{l}
u_{0} \\
u_{1}
\end{array}\right)
$$

with the latter equality holding for $u_{0} \in \mathcal{D}(A)$ and $u_{1} \in E_{1}$, and taking the form

$$
\begin{gathered}
\frac{\mathrm{d}}{\mathrm{d} t}\left(\begin{array}{cc}
S_{00}(t) & S_{01}(t) \\
S_{10}(t) & S_{11}(t)
\end{array}\right)\left(\begin{array}{l}
u_{0} \\
u_{1}
\end{array}\right)=\left(\begin{array}{cc}
0 & 1 \\
a^{-1}(A-c) & -a^{-1} b
\end{array}\right)\left(\begin{array}{cc}
S_{00}(t) & S_{01}(t) \\
S_{10}(t) & S_{11}(t)
\end{array}\right)\left(\begin{array}{l}
u_{0} \\
u_{1}
\end{array}\right)= \\
=\left(\begin{array}{ll}
S_{00}(t) & S_{01}(t) \\
S_{10}(t) & S_{11}(t)
\end{array}\right)\left(\begin{array}{cc}
0 & 1 \\
a^{-1}(A-c) & -a^{-1} b
\end{array}\right)\left(\begin{array}{l}
u_{0} \\
u_{1}
\end{array}\right)
\end{gathered}
$$

Вестник ЮУрГУ. Серия «Математическое моделирование 
we deduce the inclusions $S_{00} D(A) \subset D(A), S_{01} E_{1} \subset D(A), S_{10} D(A) \subset E_{1}$ and $S_{11} E_{1} \subset$ $E_{1}$, and equalities

$$
\begin{aligned}
& \frac{\mathrm{d} S_{00} u_{0}}{\mathrm{~d} t}=S_{10} u_{0}=S_{01} a^{-1}(A-c) u_{0}, \\
& \frac{\mathrm{d} S_{01} u_{1}}{\mathrm{~d} t}=S_{11} u_{1}=S_{00} u_{1}-\frac{b}{a} S_{01} u_{1}, \\
& \frac{\mathrm{d} S_{10} u_{0}}{\mathrm{~d} t}=a^{-1}(A-c) S_{00} u_{0}-\frac{b}{a} S_{10} u_{0}=S_{11} a^{-1}(A-c) u_{0}, \\
& \frac{\mathrm{d} S_{11} u_{1}}{\mathrm{~d} t}=a^{-1}(A-c) S_{01} u_{1}-\frac{b}{a} S_{11} u_{1}=S_{10} u_{1}-\frac{b}{a} S_{11} u_{1} .
\end{aligned}
$$

If $u_{0} \in \mathcal{D}(A)$, then it follows from (3) and (6) applied with $u_{1}=u_{0}$ that

$$
S_{01} a^{-1}(A-c) u_{0}=a^{-1}(A-c) S_{01} u_{0}=S_{10} u_{0}
$$

On the other hand, by (4), (5) and (7) it is clear that if $S_{00}$ is extended by continuity to an operator in $\mathcal{L}\left(E_{0}, E_{0}\right)$, which is possible by (4), then, for $u_{0} \in D(A)$, we have

$$
S_{00} A u_{0}=A S_{00} u_{0}
$$

By (3) and (5), we see that for $u_{0} \in D(A)$ the function $u(t)=S_{00}(t) u_{0}$ satisfies the equation

$$
\frac{\mathrm{d} u(t)}{\mathrm{d} t}=b^{-1}(A-c) u(t)-S_{11}(t) b^{-1}(A-c) u_{0} .
$$

Since $u(0)=u_{0}$, it follows that

$$
S_{00}(t) u_{0}=\exp \left(\frac{t}{b}(A-c)\right) u_{0}-\int_{0}^{t} \exp \left(\frac{t-\tau}{b}(A-c)\right) S_{11}(\tau) b^{-1}(A-c) u_{0} \mathrm{~d} \tau
$$

To simplify notation, let

$$
U(t, a, b, c, A)=\int_{0}^{t} \exp \left(\frac{t-\tau}{b}(A-c)\right) S_{11}(\tau, a, b, c, A) \mathrm{d} \tau .
$$

In view of (9), the difference between the solution

$$
u_{a}(t)=S_{00}(t, a, b, c, A) u_{0}+S_{01}(t, a, b, c, A) u_{1}
$$

to problem $\left(\mathrm{T}^{*}\right)$ and the solution

$$
u(t)=\exp \left(\frac{t}{b}(A-c)\right) u_{0}
$$

to problem $\left(D^{*}\right)$ satisfies the estimate

$$
\left\|u_{a}(t)-u(t)\right\|_{E_{0}} \leq\left\|U(t, a, b, c, A) b^{-1}(A-c) u_{0}\right\|_{E_{0}}+\left\|S_{01}(t, a, b, c, A) u_{1}\right\|_{E_{0}}
$$


for $u_{0} \in D(A), u_{1} \in E_{1}$ and $t \geq 0$. We will also obtain a similar estimate for the difference $\frac{\mathrm{d} u_{0}(t)}{\mathrm{d} t}-\frac{\mathrm{d} u(t)}{\mathrm{d} t}$. Since $S_{11}(0) u_{1}=u_{1},(6)$ implies that

$$
S_{11}(t) u_{1}=\mathrm{e}^{-\frac{b}{a} t} u_{1}+\int_{0}^{t} \mathrm{e}^{-\frac{b}{a}(t-\tau)} S_{10}(\tau) u_{1} \mathrm{~d} \tau .
$$

If $u_{1} \in D(A)$, then by (3) we may write

$$
S_{11}(t) u_{1}=\mathrm{e}^{-\frac{b}{a} t} u_{1}+\frac{b}{a} \int_{0}^{t} \mathrm{e}^{-\frac{b}{a}(t-\tau)} S_{01}(\tau) b^{-1}(A-c) u_{1} \mathrm{~d} \tau .
$$

If $u_{0} \in D\left(A^{2}\right)$ and $u_{1} \in D(A)$, then, by (3) and (4),

$$
\frac{\mathrm{d} u_{a}(t)}{\mathrm{d} t}-\frac{\mathrm{d} u(t)}{\mathrm{d} t}-\mathrm{e}^{-\frac{b}{a} t}\left(u_{1}-b^{-1}(A-c) u_{0}\right)
$$

equals

$$
\begin{gathered}
S_{10}(t) u_{0}+S_{11}(t) b^{-1}(A-c) u_{0}-\exp \left(\frac{t}{b}(A-c)\right) b^{-1}(A-c) u_{0}+ \\
+\left(S_{11}(t)-\mathrm{e}^{-\frac{b}{a} t}\right)\left(u_{1}-b^{-1}(A-c) u_{0}\right)
\end{gathered}
$$

which in turn, given that, by (5) and (8),

$$
S_{10}(t) u_{0}+S_{11}(t) b^{-1}(A-c) u_{0}=b^{-1}(A-c) S_{00}(t) u_{0}=S_{00}(t) b^{-1}(A-c) B u_{0},
$$

is equal to

$S_{00}(t) b^{-1}(A-c) u_{0}-\exp \left(\frac{t}{b}(A-c)\right) b^{-1}(A-c) u_{0}+\left(S_{11}(t)-\mathrm{e}^{-\frac{b}{a} t}\right)\left(u_{1}-b^{-1}(A-c) u_{0}\right)$,

and this, in view of (9) and (11), finally equals

$$
\begin{gathered}
-U(t, a, b, c, A)\left[b^{-1}(A-c)\right]^{2} u_{0}+ \\
+\frac{b}{a} \int_{0}^{t} \mathrm{e}^{-\frac{b}{a}(t-\tau)} S_{01}(\tau, a, b, c, A) b^{-1}(A-c)\left[u_{1}-b^{-1}(A-c) u_{0}\right] \mathrm{d} \tau .
\end{gathered}
$$

Hence, under the assumption that $u_{0} \in D\left(A^{2}\right)$ and $u_{1} \in D(A)$, we obtain the following estimate:

$$
\begin{gathered}
\left\|\frac{\mathrm{d} u_{a}(t)}{\mathrm{d} t}-\frac{\mathrm{d} u(t)}{\mathrm{d} t}-\mathrm{e}^{-\frac{b}{a} t}\left(u_{1}-b^{-1}(A-c) u_{0}\right)\right\|_{E_{0}} \leq \\
\leq\left\|U(t, a, b, c, A)\left[b^{-1}(A-c)\right]^{2} u_{0}\right\|_{E_{0}}+ \\
+\sup _{0 \leq \tau \leq t}\left\|S_{01}(\tau, a, b, c, A) b^{-1}(A-c)\left[u_{1}-b^{-1}(A-c) u_{0}\right]\right\|_{E_{0}} .
\end{gathered}
$$

In view of inequalities (10) and (12), the problem of estimating the difference $u_{a}(t)-$ $u(t)$ reduces to estimating the norms of operator-valued functions $U(t, a, b, c, A)$ and 
$S_{11}(t, a, b, c, A)$. The case of $U$ will not be treated directly, but will be tackled by means of the auxiliary function $V$ defined by

$$
V(t, a, b, c, A)=U(t, a, b, c, A)+\frac{b-\sqrt{\Delta}}{2 a} \int_{0}^{t} U(\tau, a, b, c, A) \mathrm{d} \tau .
$$

Viewing (13) as a Volterra-type equation with $U$ as an unknown function, we easily deduce that

$$
U(t, a, b, c, A)=V(t, a, b, c, A)+\frac{\sqrt{\Delta}-b}{2 a} \int_{0}^{t} \mathrm{e}^{\frac{\sqrt{\Delta}-b}{2 a}(t-\tau)} V(\tau, a, b, c, A) \mathrm{d} \tau .
$$

Since $\frac{\sqrt{\Delta}-b}{2 a}<0,(14)$ implies immediately that

$$
\|U(t, a, b, c, A)\| \leq 2 \sup _{0 \leq \tau \leq t}\|V(t, a, b, c, A)\|
$$

However, this inequality will not be used in the context of the telegraph equation $(*)$ which is of main interest to us, as in this case other, more subtle estimates are available and will be exploited that result from special properties of $V$.

The following theorem is the key to estimating the norms of operator-valued functions $V(t, a, b, c, A)$ and $S_{01}(t, a, b, c, A)$.

Comparison Theorem. Let $a>0, b>0$ and $c \geq 0$ be such that $\Delta=b^{2}-4 a c>0$, and let $k \geq 0$. Let $S_{i j}\left(t, a, b, c, k^{2}\right)$ be the real functions defined via the equality

$$
\left(\begin{array}{ll}
S_{00}\left(t, a, b, c, k^{2}\right) & S_{01}\left(t, a, b, c, k^{2}\right) \\
S_{10}\left(t, a, b, c, k^{2}\right) & S_{11}\left(t, a, b, c, k^{2}\right)
\end{array}\right)=\exp \left(t\left(\begin{array}{cc}
0 & 1 \\
\frac{k^{2}-c}{a} & -\frac{b}{a}
\end{array}\right)\right)
$$

and let (compare (13))

$$
V\left(t, a, b, c, k^{2}\right):=\mathrm{e}^{\frac{k^{2}-c}{b} t} * S_{11}\left(t, a, b, c, k^{2}\right)+\frac{b-\sqrt{\Delta}}{2 a} * \mathrm{e}^{\frac{k^{2}-c}{b} t} * S_{11}\left(t, a, b, c, k^{2}\right),
$$

where $*$ denotes convolution on the half-line $t \geq 0$. If

$$
\|\xi(t)\|_{L\left(E_{0}, E_{0}\right)} \leq M \cosh (k t), \quad-\infty<t<\infty
$$

with $M=$ const $\geq 1$, then

$$
\begin{aligned}
\left\|\exp \left(\frac{t}{b}(A-c)\right)\right\|_{L\left(E_{0}, E_{0}\right)} & \leq M \exp \left(\frac{t}{b}\left(k^{2}-c\right)\right), & & t \geq 0, \\
\left\|S_{00}(t, a, b, c, A)\right\|_{L\left(E_{0}, E_{0}\right)} & \leq M S_{00}\left(t, a, b, c, k^{2}\right), & & t \geq 0, \\
\left\|S_{01}(t, a, b, c, A)\right\|_{L\left(E_{0}, E_{0}\right)} & \leq M S_{01}\left(t, a, b, c, k^{2}\right), & & t \geq 0,
\end{aligned}
$$

and

$$
\|V(t, a, b, c, A)\|_{L\left(E_{0}, E_{0}\right)} \leq M V\left(t, a, b, c, k^{2}\right), \quad t \geq 0 .
$$




\section{Proof of the Comparison Theorem}

By (2), we have

$$
\|\exp (t A)\| \leq \frac{1}{2 \sqrt{\pi t}} \int_{-\infty}^{\infty} \mathrm{e}^{-\frac{\tau^{2}}{4 t}}\|\xi(\tau)\| \mathrm{d} \tau \leq M \frac{1}{2 \sqrt{\pi t}} \int_{-\infty}^{\infty} \mathrm{e}^{-\frac{\tau^{2}}{4 t}} \cosh (k t) \mathrm{d} \tau=M \exp \left(t k^{2}\right)
$$

and so

$$
\left\|\exp \left(\frac{t}{b}(A-c)\right)\right\| \leq \mathrm{e}^{-\frac{c}{b} t}\|\exp (t A)\| \leq M \mathrm{e}^{-\frac{c}{b} t} \mathrm{e}^{\frac{t}{b} k^{2}}=M \mathrm{e}^{\frac{k^{2}-c}{b} t} .
$$

Similarly, by (1),

$$
\begin{aligned}
& \left\|S_{01}(t, a, b, c, A)\right\| \leq \int_{0}^{t} K(t, \tau)\left\|\xi\left(\frac{\tau}{\sqrt{a}}\right)\right\| \mathrm{d} \tau \leq \\
\leq & M \int_{0}^{t} K(t, \tau) \cosh \left(\frac{k \tau}{\sqrt{a}}\right) \mathrm{d} \tau=M S_{01}\left(t, a, b, c, k^{2}\right),
\end{aligned}
$$

because the kernel $K(t, \tau)=\mathrm{e}^{-\frac{b}{2 a} t} J_{0}\left(i \frac{\sqrt{\Delta}}{2 a} \sqrt{\tau^{2}-t^{2}}\right)$ is non-negative for $0 \leq \tau \leq t$. Inequalities (16) and (18) are thus proved. Inequality (17) is established in a similar manner. The proof of (19) is more complicated. According to a well-known result from the theory of semigroups of operators, there is a constant $\lambda_{0}$ such that for $\lambda>\lambda_{0}$ we have

$$
b(b \lambda+c-A)^{-1}=\left(\lambda-b^{-1}(A-c)\right)^{-1}=\int_{0}^{\infty} \mathrm{e}^{-\lambda t} \exp \left(\frac{t}{b}(A-c)\right) \mathrm{d} t
$$

and

$$
\left(\lambda-B_{a, b, c}\right)^{-1}=\int_{0}^{\infty} \mathrm{e}^{-\lambda t} \exp \left(t B_{a, b, c}\right) \mathrm{d} t .
$$

Since (15) implies that

$$
\lambda\left(\lambda^{2}-A\right)^{-1}=\int_{0}^{\infty} \mathrm{e}^{-\lambda t} \xi(t) \mathrm{d} t \quad \text { for } \lambda>k
$$

it follows that for $\lambda$ so large that $a \lambda^{2}+b \lambda+c>k^{2}$ we have

$$
\begin{gathered}
\left(\lambda-B_{a, b, c}\right)^{-1}=\left(\lambda-\left(\begin{array}{cc}
0 & 1 \\
a^{-1}(A-c) & -a^{-1} b
\end{array}\right)\right)^{-1}= \\
=\left(\begin{array}{cc}
(a \lambda+b)\left(a \lambda^{2}+b \lambda+c-A\right)^{-1} & a\left(a \lambda^{2}+b \lambda+c-A\right)^{-1} \\
(A-c)\left(a \lambda^{2}+b \lambda+c-A\right)^{-1} & a \lambda\left(a \lambda^{2}+b \lambda+c-A\right)^{-1}
\end{array}\right) .
\end{gathered}
$$

This together with (21) implies the existence of a constant $\lambda_{1}$ such that

$$
\int_{0}^{\infty} \mathrm{e}^{-\lambda t} S_{11}(t, a, b, c, A)=a \lambda\left(a \lambda^{2}+b \lambda+c-A\right)^{-1} \text { for } \lambda>\lambda_{1} .
$$


Since the Laplace transform of the convolution of two functions (on the half-line $0 \leq t<$ $\infty)$ is the product of the transforms of these functions, equality (13) written as

$$
\begin{gathered}
V(t, a, b, c, A)=\exp \left(\frac{t}{b}(A-c)\right) * S_{11}(t, a, b, c, A)+ \\
+\frac{b-\sqrt{\Delta}}{2 a} * \exp \left(\frac{t}{b}(A-c)\right) * S_{11}(t, a, b, c, A)
\end{gathered}
$$

and combined with (20) and (23) implies that

$$
\int_{0}^{\infty} \mathrm{e}^{-\lambda t} V(t, a, b, c, A) \mathrm{d} t=a\left(\lambda+\frac{b-\sqrt{\Delta}}{2 a}\right)\left(\lambda-b^{-1}(A-c)\right)^{-1}\left(a \lambda^{2}+b \lambda+c-A\right)^{-1}
$$

for $\lambda>\lambda_{2}=\max \left(\lambda_{0}, \lambda_{1}\right)$. Introducing the functions

$$
\begin{gathered}
g_{1}(\lambda)=\left(\lambda+\frac{c}{b}\right)^{\frac{1}{2}}, \\
g_{2}(\lambda)=\left(a \lambda^{2}+b \lambda+c\right)^{\frac{1}{2}}, \\
h(\lambda)=a \frac{\lambda+\frac{b-\sqrt{\Delta}}{2 a}}{\left(\lambda+\frac{c}{b}\right)^{\frac{1}{2}}\left(a \lambda^{2}+b \lambda+c\right)^{\frac{1}{2}}},
\end{gathered}
$$

we may rewrite the last identity as

$$
\int_{0}^{\infty} \mathrm{e}^{-\lambda t} V(t, a, b, c, A) \mathrm{d} t=h(\lambda) g_{1}(\lambda)\left(\left[g_{1}(\lambda)\right]^{2}-A\right)^{-1} g_{2}(\lambda)\left(\left[g_{2}(\lambda)\right]^{2}-A\right)^{-1}
$$

for $\lambda>\lambda_{2}$. But now, by (22), we see that

$$
\begin{gathered}
\int_{0}^{\infty} \mathrm{e}^{-\lambda t} V(t, a, b, c, A) \mathrm{d} t=h(\lambda) \int_{0}^{\infty} \mathrm{e}^{-g_{1}(\lambda) t} \xi(t) \mathrm{d} t \int_{0}^{\infty} \mathrm{e}^{-g_{2}(\lambda) s} \xi(s) \mathrm{d} s= \\
=h(\lambda) \int_{0}^{\infty} \int_{0}^{\infty} \mathrm{e}^{-g_{1}(\lambda) t-g_{2}(\lambda) s} \frac{1}{2}[\xi(t+s)+\xi(t-s)] \mathrm{d} t \mathrm{~d} s
\end{gathered}
$$

for $\lambda>\lambda_{2}$. Analogously,

$$
\int_{0}^{\infty} \mathrm{e}^{-\lambda t} V\left(t, a, b, c, k^{2}\right) \mathrm{d} t=h(\lambda) \int_{0}^{\infty} \int_{0}^{\infty} \mathrm{e}^{-g_{1}(\lambda) t-g_{2}(\lambda) s} \frac{1}{2}[\cosh (t+s)+\cosh (t-s)] \mathrm{d} t \mathrm{~d} s .
$$

Thus, for each $u \in E_{0}$ such that $\|u\|=1$ and each bounded linear functional $f$ on $E_{0}$ such that $\|f\|=1$, we have

$$
\begin{gathered}
\int_{0}^{\infty} \mathrm{e}^{-\lambda t}\left[M V\left(t, a, b, c, k^{2}\right)-\langle f, V(t, a, b, c, A) u\rangle\right] \mathrm{d} t= \\
=h(\lambda) \int_{0}^{\infty} \int_{0}^{\infty} \mathrm{e}^{-g_{1}(\lambda) t-g_{2}(\lambda) s} \Psi(t, s) \mathrm{d} t \mathrm{~d} s
\end{gathered}
$$


for $\lambda>\lambda_{2}$, while, by (15), the function

$$
\Psi(t, s)=\frac{1}{2} M[\cosh (t+s)+\cosh (t-s)]-\frac{1}{2}\langle f,[\xi(t+s)+\xi(t-s)] u\rangle
$$

is non-negative. Let

$$
F(\lambda)=h(\lambda) \int_{0}^{\infty} \int_{0}^{\infty} \mathrm{e}^{-g_{1}(\lambda) t-g_{2}(\lambda) s} \Psi(t, s) \mathrm{d} t \mathrm{~d} s
$$

and let us assume for now that

the function $F(-\lambda)$ is completely monotone in the interval $-\infty<\lambda<-\lambda_{2}$,

i.e., that $\frac{\mathrm{d}^{n} F(-\lambda)}{\mathrm{d} \lambda^{n}}>0$ for all $n=0,1, \ldots$ and $\lambda \in\left(-\infty,-\lambda_{2}\right)$.

It follows (26) and (27), by virtue of the Post-Widder formula for inversion of the Laplace transform, that

$$
M V\left(t, a, b, c, k^{2}\right)-\langle f, V(t, a, b, c, A) u\rangle=\left.\lim _{n \rightarrow \infty} \frac{(-1)^{n}}{(n-1) !}\left(\frac{n}{t}\right)^{n} \frac{\mathrm{d}^{n-1} F(\lambda)}{\mathrm{d} \lambda^{n-1}}\right|_{\lambda=\frac{n}{t}} \geq 0
$$

for each $t>0$. Since the only restriction imposed on $u$ and $f$ was $\|u\|=\|f\|=1$, this results in inequality (19).

The idea of exploiting the Post-Widder formula to obtain estimates as above is taken from the paper by M. Sova [9], who was the first to study the asymptotic behaviour, as $a \rightarrow 0$, of solutions of initial-value problem of type $\left(\mathrm{T}^{*}\right)$ in a non-Hilbert space. The idea to use completely monotone functions is due to the author of the present paper; this approach simplifies the analysis and sharpens the estimates.

We now present the proof of (27). It suffices to show that

$$
\begin{array}{ll}
\frac{\mathrm{d}^{n}\left[-g_{1}(-\lambda)\right]}{\mathrm{d} \lambda^{n}}>0 & \text { for } n=0,1, \ldots \text { and } \lambda \in\left(-\infty,-\lambda_{2}\right), \\
\frac{\mathrm{d}^{n}\left[-g_{2}(-\lambda)\right]}{\mathrm{d} \lambda^{n}}>0 & \text { for } n=0,1, \ldots \text { and } \lambda \in\left(-\infty,-\lambda_{2}\right),
\end{array}
$$

and

$$
\frac{\mathrm{d}^{n} h(-\lambda)}{\mathrm{d} \lambda^{n}}>0 \quad \text { for } n=0,1, \ldots \text { and } \lambda \in\left(-\infty,-\lambda_{2}\right)
$$

That (a) is true follows immediately from the formula

$$
\frac{\mathrm{d}^{n}\left[-g_{1}(-\lambda)\right]}{\mathrm{d} \lambda^{n}}=-\frac{\mathrm{d}^{n}}{\mathrm{~d} \lambda^{n}}\left(\frac{c}{b}-\lambda\right)^{\frac{1}{2}}=\frac{1}{2} \frac{1}{2} \frac{3}{2} \ldots \frac{2 n-3}{2}\left(\frac{c}{b}-\lambda\right)^{-\frac{2 n-1}{2}} .
$$

For the proof of (b) we calculate, for $\lambda<0$,

$$
\frac{\mathrm{d}}{\mathrm{d} \lambda}\left[-g_{2}(-\lambda)\right]=-\frac{\mathrm{d}}{\mathrm{d} \lambda}\left(a \lambda^{2}-b \lambda+c\right)^{\frac{1}{2}}=\left(\frac{b}{2}-a \lambda\right)\left(a \lambda^{2}-b \lambda+c\right)^{-\frac{1}{2}}>0
$$


and

$$
\begin{gathered}
\frac{\mathrm{d}^{2}}{\mathrm{~d} \lambda^{2}}\left[-g_{2}(-\lambda)\right]=-a\left(a \lambda^{2}-b \lambda+c\right)^{-\frac{1}{2}}+\left(\frac{b}{2}-a \lambda\right)^{2}\left(a \lambda^{2}-b \lambda+c\right)^{-\frac{3}{2}}= \\
=\frac{\Delta}{4}\left(a \lambda^{2}-b \lambda+c\right)^{-\frac{3}{2}}=\frac{\Delta}{4} a^{-\frac{3}{2}}\left(\mu_{1}-\lambda\right)^{-\frac{3}{2}}\left(\mu_{2}-\lambda\right)^{-\frac{3}{2}}>0,
\end{gathered}
$$

where $\mu_{1}=\frac{b-\sqrt{\Delta}}{2 a}>0$ and $\mu_{2}=\frac{b+\sqrt{\Delta}}{2 a}>0$. Since

$$
\frac{\mathrm{d}^{k}}{\mathrm{~d} \lambda^{k}}\left(\mu_{j}-\lambda\right)^{-\frac{3}{2}}=\frac{3}{2} \cdot \frac{5}{2} \cdots \frac{2 k+1}{2}\left(\mu_{j}-\lambda\right)^{-\frac{2 k+3}{2}}
$$

for $k=0,1, \ldots, j=1,2$ and $\lambda<0$, Leibniz's formula implies that

$$
\frac{\mathrm{d}^{2+n}\left[-g_{2}(-\lambda)\right]}{\mathrm{d} \lambda^{2+n}}=\frac{\Delta}{4} a^{-\frac{3}{2}} \sum_{k=0}^{n}\left(\begin{array}{l}
n \\
k
\end{array}\right) \frac{\mathrm{d}^{k}\left(\mu_{1}-\lambda\right)^{-\frac{3}{2}}}{\mathrm{~d} \lambda^{k}} \frac{\mathrm{d}^{n-k}\left(\mu_{2}-\lambda\right)^{-\frac{3}{2}}}{\mathrm{~d} \lambda^{n-k}}>0
$$

for $\lambda<0$ and $n=1,2, \ldots$

We are left with showing (c). To this end, we observe that

$$
h(-\lambda)=a \frac{\mu_{1}-\lambda}{\left(\frac{c}{a}-\lambda\right)^{\frac{1}{2}} a^{\frac{1}{2}}\left(\mu_{1}-\lambda\right)^{\frac{1}{2}}\left(\mu_{2}-\lambda\right)^{\frac{1}{2}}}=a^{\frac{1}{2}}\left(\frac{\mu_{1}-\lambda}{\frac{c}{b}-\lambda}\right)^{\frac{1}{2}}\left(\mu_{2}-\lambda\right)^{-\frac{1}{2}} .
$$

Since

$$
\frac{\mathrm{d}^{k}}{\mathrm{~d} \lambda^{k}}\left(\mu_{2}-\lambda\right)^{-\frac{1}{2}}=\frac{1}{2} \cdot \frac{3}{2} \cdots \frac{2 k-1}{2}\left(\mu_{2}-\lambda\right)^{-\frac{2 k+1}{2}}
$$

for $\lambda<0$ and $k=1,2, \ldots$, Leibniz's formula shows that our task reduces to proving that

$$
\frac{\mathrm{d}^{k}}{\mathrm{~d} \lambda^{k}}\left(\frac{\mu_{1}-\lambda}{\frac{c}{b}-\lambda}\right)^{\frac{1}{2}}>0 \quad \text { for } \lambda<0 \text { and } k=0,1, \ldots \text {. }
$$

Inequality $\left(\mathrm{c}^{*}\right)$ results from

$$
D:=\lambda_{1}-\frac{c}{b}=\frac{b-\sqrt{\Delta}}{2 a}-\frac{c}{b} \geq 0
$$

since $b^{2}-2 a c \geq b \sqrt{\Delta}$; the latter condition may easily be checked by taking squares of both sides. By (28), we have

$$
\begin{aligned}
\frac{\mathrm{d}}{\mathrm{d} \lambda}\left(\frac{\mu_{1}-\lambda}{\frac{c}{b}-\lambda}\right)^{\frac{1}{2}}= & \frac{\mathrm{d}}{\mathrm{d} \lambda}\left(\frac{\frac{c}{b}+D-\lambda}{\frac{c}{b}-\lambda}\right)^{\frac{1}{2}}=\frac{1}{2}\left(\frac{\frac{c}{b}-\lambda}{\frac{c}{b}+D-\lambda}\right)^{\frac{1}{2}} \frac{D}{\left(\frac{c}{b}-\lambda\right)^{2}}= \\
& =\frac{D}{2}\left(\frac{c}{b}+D-\lambda\right)^{-\frac{1}{2}}\left(\frac{c}{b}-\lambda\right)^{-\frac{3}{2}}
\end{aligned}
$$

and thus we see that inequality $\left(\mathrm{c}^{*}\right)$ is true for $k=0,1$. To prove it for $k=2,3, \ldots$, one needs to apply Leibniz's formula in a way similar to that used in our proof of (b). Hence, inequality (c) is seen to be true. The proof of the comparison theorem is thereby completed. 


\section{Consequences of the Comparison Theorem in the Case $k=0$}

We have

$$
\begin{gathered}
V(t, a, b, c, 0)=\mathrm{e}^{-\frac{c}{b} t} *\left(S_{11}(t, a, b, c, 0)+\frac{b-\sqrt{\Delta}}{2 a} \int_{0}^{t} S_{11}(\tau, a, b, c, 0) \mathrm{d} \tau\right)= \\
=\mathrm{e}^{-\frac{c}{b} t} *\left(S_{11}(t, a, b, c, 0)+\frac{b-\sqrt{\Delta}}{2 a} S_{01}(t, a, b, c, 0)\right)=\mathrm{e}^{-\frac{c}{b} t} * u(t),
\end{gathered}
$$

where

$$
u(t)=S_{11}(t, a, b, c, 0)+\frac{b-\sqrt{\Delta}}{2 a} S_{01}(t, a, b, c, 0)
$$

is the solution of the equation

$$
a u^{\prime \prime}+b u^{\prime}+c u=0
$$

with initial conditions

$$
\begin{gathered}
u(0)=1, \\
u^{\prime}(0)=S_{11}^{\prime}(0)+\frac{b-\sqrt{\Delta}}{2 a}=-\frac{b}{a}+\frac{b-\sqrt{\Delta}}{2 a}=-\frac{b+\sqrt{\Delta}}{2 a} .
\end{gathered}
$$

Therefore we have

$$
u(t)=\mathrm{e}^{-\frac{b+\sqrt{\Delta}}{2 a} t}
$$

and so

$$
\begin{gathered}
V(t, a, b, c, 0)=\mathrm{e}^{-\frac{c}{b} t} \int_{0}^{t} \mathrm{e}^{\left(\frac{c}{b}-\frac{b+\sqrt{\Delta}}{2 a}\right) \tau} \mathrm{d} \tau=\mathrm{e}^{-\frac{c}{b} t} \int_{0}^{t} \mathrm{e}^{-\left(D+\frac{\sqrt{\Delta}}{a}\right) \tau} \mathrm{d} \tau \leq \\
\leq \mathrm{e}^{-\frac{c}{b} t} \frac{1}{D+\frac{\sqrt{\Delta}}{a}} \leq \frac{a}{\sqrt{\Delta}} \mathrm{e}^{-\frac{c}{b} t} .
\end{gathered}
$$

Also

$$
\begin{gathered}
\int_{0}^{t} \mathrm{e}^{\frac{\sqrt{\Delta}-b}{2 a}(t-\tau)} V(\tau, a, b, c, 0) \mathrm{d} \tau=\int_{0}^{t} \mathrm{e}^{\frac{\sqrt{\Delta}-b}{2 a} \tau} V(t-\tau, a, b, c, 0) \mathrm{d} \tau \leq \\
\leq \frac{a}{\sqrt{\Delta}} \int_{0}^{t} \mathrm{e}^{\frac{\sqrt{\Delta}-b}{2 a} \tau} \mathrm{e}^{-\frac{c}{b}(t-\tau)} \mathrm{d} \tau=\frac{a}{\sqrt{\Delta}} \mathrm{e}^{-\frac{c}{b} t} \int_{0}^{t} \mathrm{e}^{-D \tau} \mathrm{d} \tau .
\end{gathered}
$$

Assume that inequality (15) holds with $k=0$. Then, in view of the above, inequality (19) and equality (14), we obtain the estimate

$$
\begin{gathered}
\|U(t, a, b, c, A)\|_{L\left(E_{0}, E_{0}\right)} \leq \mathrm{e}^{-\frac{c}{b} t} M \frac{a}{\sqrt{\Delta}}\left[1+\frac{b-\sqrt{\Delta}}{2 a} \int_{0}^{t} \mathrm{e}^{-D \tau} \mathrm{d} \tau\right] \leq \\
\leq \mathrm{e}^{-\frac{c}{b} t} M \frac{a}{\sqrt{\Delta}}\left(1+\frac{c}{\sqrt{\Delta}} t\right),
\end{gathered}
$$

for we have $\frac{b-\sqrt{\Delta}}{2 a} \leq \frac{c}{\sqrt{\Delta}}$. Since $u(t)=S_{01}(t, a, b, c, 0)$ is the solution of the equation $a u^{\prime \prime}+b u^{\prime}+c u=0$ satisfying the conditions $u(0)=0$ and $u^{\prime}(0)=1$, we have 
$S_{01}(t, a, b, c, 0)=\left(\nu_{1}-\nu_{2}\right)^{-1}\left(\mathrm{e}^{\nu_{1} t}-\mathrm{e}^{\nu_{2} t}\right)$, where $\nu_{1}=\frac{-b+\sqrt{\Delta}}{2 a}$ and $\nu_{2}=\frac{-b-\sqrt{\Delta}}{2 a}$, which implies that $S_{01}(t, a, b, c, 0) \leq \frac{a}{\sqrt{\Delta}}$ for $t \geq 0$. In view of inequality (18), we thus have

$$
\left\|S_{01}(t, a, b, c, A)\right\|_{L\left(E_{0}, E_{0}\right)} \leq M \frac{a}{\sqrt{\Delta}} \text { for } t \geq 0 .
$$

Combining together (10), (12), (29) and (30), we finally obtain the following estimates of the difference between the solution $u_{a}(t)$ of problem $\left(\mathrm{T}^{*}\right)$ and the solution $u(t)$ of problem $\left(D^{*}\right)$ :

(i) if $u_{0} \in D(A)$ and $u_{1} \in E_{1}$, then

$$
\left\|u_{a}(t)-u(t)\right\|_{E_{0}} \leq \frac{a}{\sqrt{\Delta}} M\left\{\mathrm{e}^{-\frac{c}{b} t}\left(1+\frac{c}{\sqrt{\Delta}} t\right)\left\|b^{-1}(A-c) u_{0}\right\|_{E_{0}}+\left\|u_{1}\right\|_{E_{0}}\right\}
$$

for $t \geq 0$;

(ii) and if, furthermore, $u_{0} \in D\left(A^{2}\right)$ and $u_{1} \in D(A)$, then also

$$
\begin{gathered}
\left\|\frac{\mathrm{d} u_{a}(t)}{\mathrm{d} t}-\frac{\mathrm{d} u(t)}{\mathrm{d} t}-\mathrm{e}^{-\frac{b}{a} t}\left(u_{1}-b^{-1}(A-c) u_{0}\right)\right\|_{E_{0}} \leq \\
\leq \frac{a}{\sqrt{\Delta}} M\left\{\mathrm{e}^{-\frac{c}{b} t}\left(1+\frac{c}{\sqrt{\Delta}} t\right)\left\|\left[b^{-1}(A-c)\right]^{2} u_{0}\right\|_{E_{0}}+\left\|b^{-1}(A-c)\left[u_{1}-b^{-1}(A-c) u_{0}\right]\right\|_{E_{0}}\right\}
\end{gathered}
$$

for $t \geq 0$.

For $c=0$ these inequalities are identical to those obtained in the paper [3].

\section{References}

1. Baran W. Stabilność schematów różnicowych aproksymujacych zależne od czasu równania: transportu, telegrafistów i dyfuzji. Instytut Badań Jądrowych, Zakład Obliczeń Numerycznych i Teorii Transportu, 1972. (in Polish)

2. Kisyński J. Sur les équations hyperboliques avec petit paramètre. Colloquium Mathematicum, 1963, vol. 10, no. 2, pp. 331-343. (in French) DOI: 10.4064/cm-10-2-331-343

3. Kisyński J. On a Second Order Cauchy's Problem in a Banach Space. Bulletin Polish Acad. Sci. Math. Series: Sci. Math. Astr. Phys., 1970, vol. 18, pp. 371-374.

4. Kisyński J. On Cosine Operator Functions and One-Parameter Groups of Operators. Studia Mathematica, 1972, vol. 44, no. 1, pp. 93-105. DOI: 10.4064/sm-44-1-93-105

5. Kisyński J. On the Connections between Cosine Operator Functions and One Parameter SemiGroups and Groups of Operators. Warsaw, Wydawnictwo Uniwersytetu Warszawskiego, 1972, no. 6, pp. 1-9.

6. Nedoma J. Initial Cauchy Problem for Hyperbolic Equations with Small Parameter. Časopis pro pěstováni matematiky, 1967, vol. 92, no. 4, pp. 392-417.

7. Smoller J.A. Singular Perturbations and a Theorem of Kisyński. Journal of Mathematical Analysis and Applications, 1965, vol. 12, pp. 105-114. DOI: 10.1016/0022-247X(65)90058-2

8. Sova M. Cosine Operator Functions. Rozprawy Matematyczne, 1966, vol. 49, pp. 1-47.

9. Sova M. Équations hyperboliques avec petit paramètre dans les espaces de Banach généraux. Colloquium Mathematicum, 1970, vol. 21, no. 2, pp. 303-320. (in French) DOI: 10.4064/cm21-2-303-320 
10. Weinberg A.M., Wigner E.P. The Physical Theory of Neutron Chain Reactors. Chicago, University of Chicago Press, 1958.

11. Zlámal M. On the Mixed Problem for a Hyperbolic Equation with a Small Parameter. Czechoslovak Mathematical Journal, 1959, vol. 9, no. 2, pp. 218-242. (in Russian)

12. Zlámal M. Sur l'équation des télégraphistes avec un petit paramètre. Atti Accad. Naz. Lincei. Rend. Cl. Sci. Fis. Mat. Nat., 1959, vol. 27, no. 8, pp. 324-332. (in French)

13. Zlámal M. The Mixed Problem for Hyperbolic Equations with a Small Parameter, Czechoslovak Mathematical Journal, 1960, vol. 10, no. 1, pp. 83-122. (in Russian) 